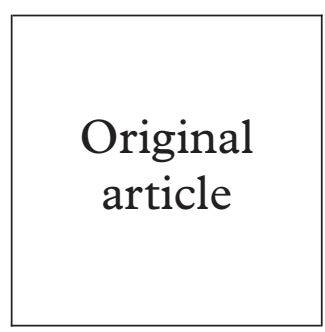

\title{
Impact of HIV on adult (15-54) mortality in London: 1979-96
}

\author{
Matthew Hickman, Martin Bardsley, Daniela De Angelis, Helen Ward
}

Objective: To describe the impact of HIV on mortality in men and women aged 15-54 in London.

Design: Combination of routine mortality statistics with reports of AIDS deaths adjusted for underreporting and change in address from time of report to time of death. Calculation of standardised mortality ratios (SMRs) for males including and excluding HIV comparing inner London and outer London with the rest of England and Wales.

Methods: Comparison of trends in all cause mortality and SMRs in males over time. Comparison of trends in HIV related deaths with other main causes of deaths in males and females in London.

Results: Age standardised rates for the rest of England and Wales showed a continual decline from 1979 to 1996 but rates in inner London males (ages 15-54) stopped declining around 1984-5 leading to a considerable increase in the SMR for inner London from 127 for 1985-7 to 171 for 1994-6. SMRs excluding HIV related deaths for inner London, however, showed no significant change over this time. There was a fall in HIV related mortality in 1996, though HIV was still the leading cause of death in males and second leading cause of death in females in inner London, and the fourth commonest cause of death in males in outer London.

Conclusion: These data are the first to indicate the impact of HIV on mortality within a significant population in England and Wales. They show that public health priorities in London are different from the rest of the country. Analyses of trends of all cause mortality in people under 65 may mislead unless they take account of HIV.

(Sex Transm Inf 1999;75:385-388)

Keywords: HIV; adults; mortality; London

\section{Introduction}

Premature mortality is one of the key indicators of the burden and public health importance of a disease. By the end of 1996, over 6 million people had died from HIV associated causes worldwide, and HIV was ranked sixth highest cause of death among young adults by the global burden of disease study. ${ }^{12}$ HIV has had the greatest impact on mortality in developing countries especially sub-Saharan Africa $^{3}$; but it has also had an effect on mortality in some urban centres of developed countries. ${ }^{4}$ In the United States, HIV was the leading cause of death among adults in many cities, ${ }^{5}$ and in Italy and parts of Spain it was a major cause of death in people aged 25-34. ${ }^{67}$

Evidence of the impact of HIV on general mortality in England and Wales was limited to suggestions that AIDS along with suicide and open verdicts contributed to an observed increase in mortality among men under $45 .{ }^{8} \mathrm{An}$ earlier study also suggested that HIV infection was one of the leading causes of death in young men in a small area of London. ${ }^{9}$ One difficulty is that HIV related deaths are partially hidden among routine mortality statistics, as they can be classified under a number of different ICD codes often without mentioning underlying HIV infection. ${ }^{10}{ }^{11}$ It has been observed that male mortality in inner London has not been falling as fast as in other parts of the country, and some of this may be related to the effects of HIV. $^{12} 13$
In 1996, for the first time, surveillance reports of AIDS related deaths declined in the United States and in the United Kingdom, probably as a result of combination therapy. ${ }^{14}$ In this paper we illustrate the impact of HIV on mortality for adults aged 15-54 resident in London from 1979 to 1996 by combining AIDS surveillance data with mortality statistics, and compare overall mortality trends with those in the rest of England and Wales.

\section{Methods}

Trends in AIDS related deaths in adults aged 15-54 to the end of 1996 were obtained from the Public Health Laboratory Surveillance, Communicable Disease Surveillance Centre. The population and numbers of deaths by cause were obtained from the Office for National Statistics (ONS). These were aggregated by area of residence, defined by local government boundaries, into inner London, outer London, rest of England and Wales, and elsewhere. Reports of AIDS deaths were adjusted for underreporting by multiplying by 1.13 (in common with other studies using AIDS reports $\left.{ }^{15}{ }^{16}\right)$; and for potential changes in address between time of report and time of death. The latter were derived from a matching exercise that linked AIDS reports to ONS death certificates in 1993 (CDSC, personal communication). This showed, for example, that of all male AIDS cases that died in 1993 and that were reported as resident in inner London, $93 \%$ also died in inner London, $2 \%$ in 


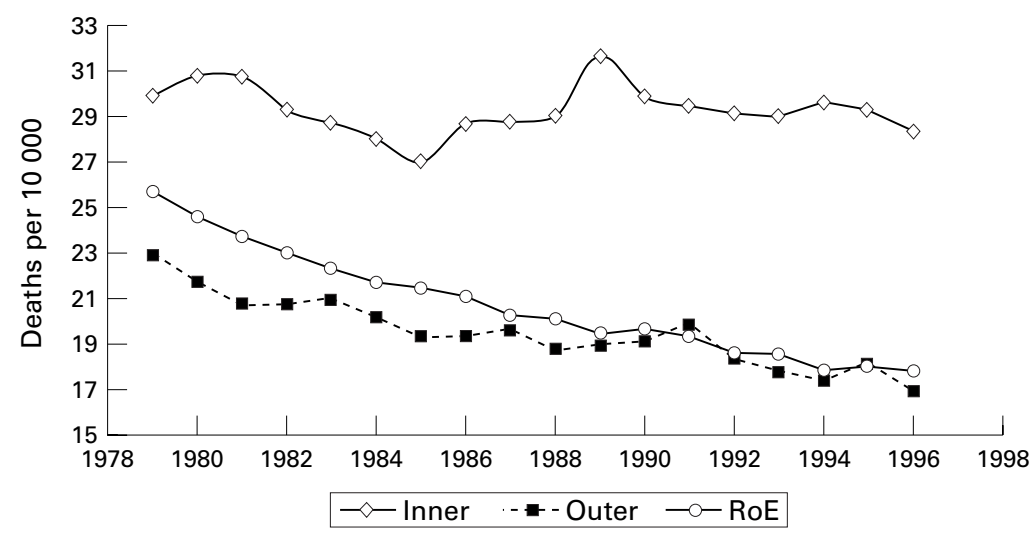

Figure 1 Trends in mortality in males aged 15-54.

outer London, $4 \%$ in rest of England and Wales, and $1 \%$ elsewhere. An additional $8 \%$ of AIDS cases reported as resident in outer London died in inner London. Thus, AIDS reports were readjusted to coincide with area of residence recorded on ONS mortality statistics.

There was no need to adjust AIDS death reports for reporting delay as sufficient time was allowed for late reports. Nor were they further adjusted to take account of the estimated $5 \%$ of HIV infected people that die pre-AIDS; we considered these would have been covered by the general adjustment for underreporting.
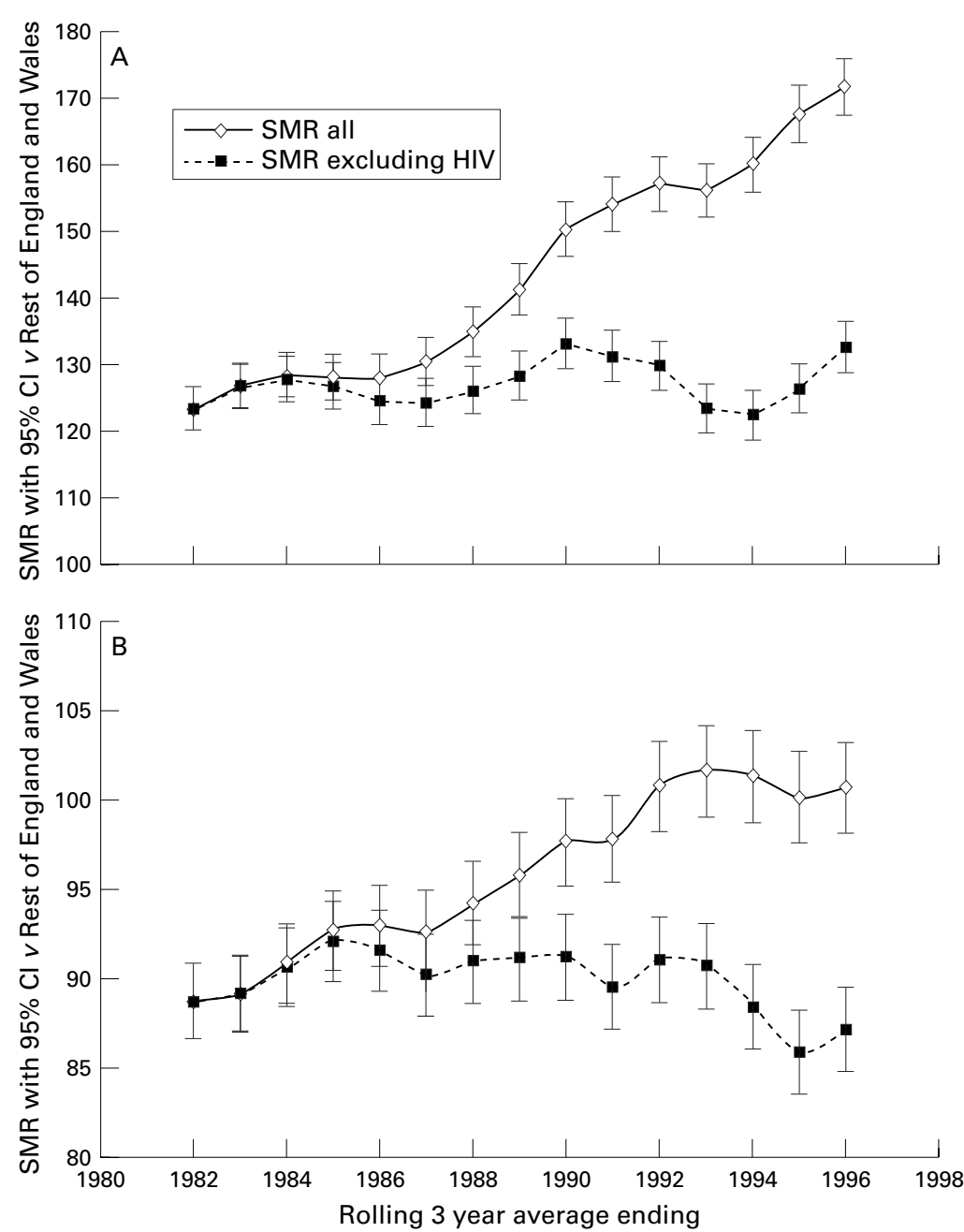

Figure 2 Standardised mortality ratio (SMR) of males aged 15-54 with and without $H I V$ related deaths $(A)$ in inner London, $(B)$ in outer London.
The impact of HIV related deaths in inner and outer London was shown in three ways. Firstly, comparisons of trends between geographical areas in male all cause mortality were made using directly standardised rates against the standard European population. Summaries of the trends were estimated using simple regression techniques applied to the log of the annual rates, and presented as an average percentage change in mortality per year.

Secondly, also for males, standardised mortality ratios (SMRs) were calculated excluding and including HIV related deaths: by subtracting them (or not) from the total observed deaths and comparing the rates with those in the rest of England and Wales (excluding or including HIV related deaths). Estimates of the number of HIV related deaths were obtained from surveillance data. Confidence intervals $(95 \%)$ for these ratios were based on the approximation $\mathrm{SMR} /\left(\mathrm{e}^{1.96 / \text { number }}\right.$ of deaths $)$ to $\operatorname{SMR}^{\star}\left(\mathrm{e}^{1.96 / \text { /number of deaths }}\right)$.

Thirdly, for males and females the contribution of the main causes of death (which were unlikely to be substitutes for underlying HIV infection) obtained from ONS mortality statistics were compared with rates of HIV related mortality over time. The conditions used were defined in ICD 9 codes as follows: ischaemic heart disease (ICD 410-414); cerebrovascular disease/stroke and hypertension (ICD 430438); lung cancer (ICD 162); suicide, self inflicted, and undetermined injury (ICD E95-E959, E980-E989); accidents (ICD E800E949); breast cancer (ICD 174); and chronic liver disease and cirrhosis (ICD 571).

\section{Results}

There was a total 9735 AIDS cases in people aged 15-54 reported as having died from 1979 to 1996 : $92 \%$ (8955) were in males; and $34 \%$ (3070) reported as resident in inner London. Over the same period and in the same age group ONS recorded a total of 718747 deaths: $63 \%$ (451 487) in males, and 10\% (46 812) resident in inner London. Trends in the observed and adjusted number of reports of AIDS deaths by sex, year, and area of residence are available on request (unpublished table).

Figure 1 shows trends in age standardised all cause mortality among males aged 15-54 for inner London, outer London, and the rest of England. Throughout the period mortality in inner London was higher than the rest of England and Wales and outer London; it also diverged markedly from the national trends from 1986 onwards. Thus, based on the linear regression of the log values, the decline in mortality between 1979 and 1985 in males aged 15-54 was similar-that is, 3\% (SE $0.2 \%$ ) in rest of England and Wales, $2.3 \%$ (SE $0.4 \%$ ) in outer London, and $2.0 \%$ (SE $0.4 \%$ ) in inner London. From 1986 to 1996 mortality declined by on average $1.6 \%$ (SE $0.1 \%$ ) in rest of England and Wales, $1.3 \%$ (SE $0.3 \%$ ) in outer London, while there was no significant change in inner London (that is, average fall $0.1 \%$, SE $0.3 \%)$.

Figure 2A shows that the 3 year average SMR (relative to rates for that time in the rest 

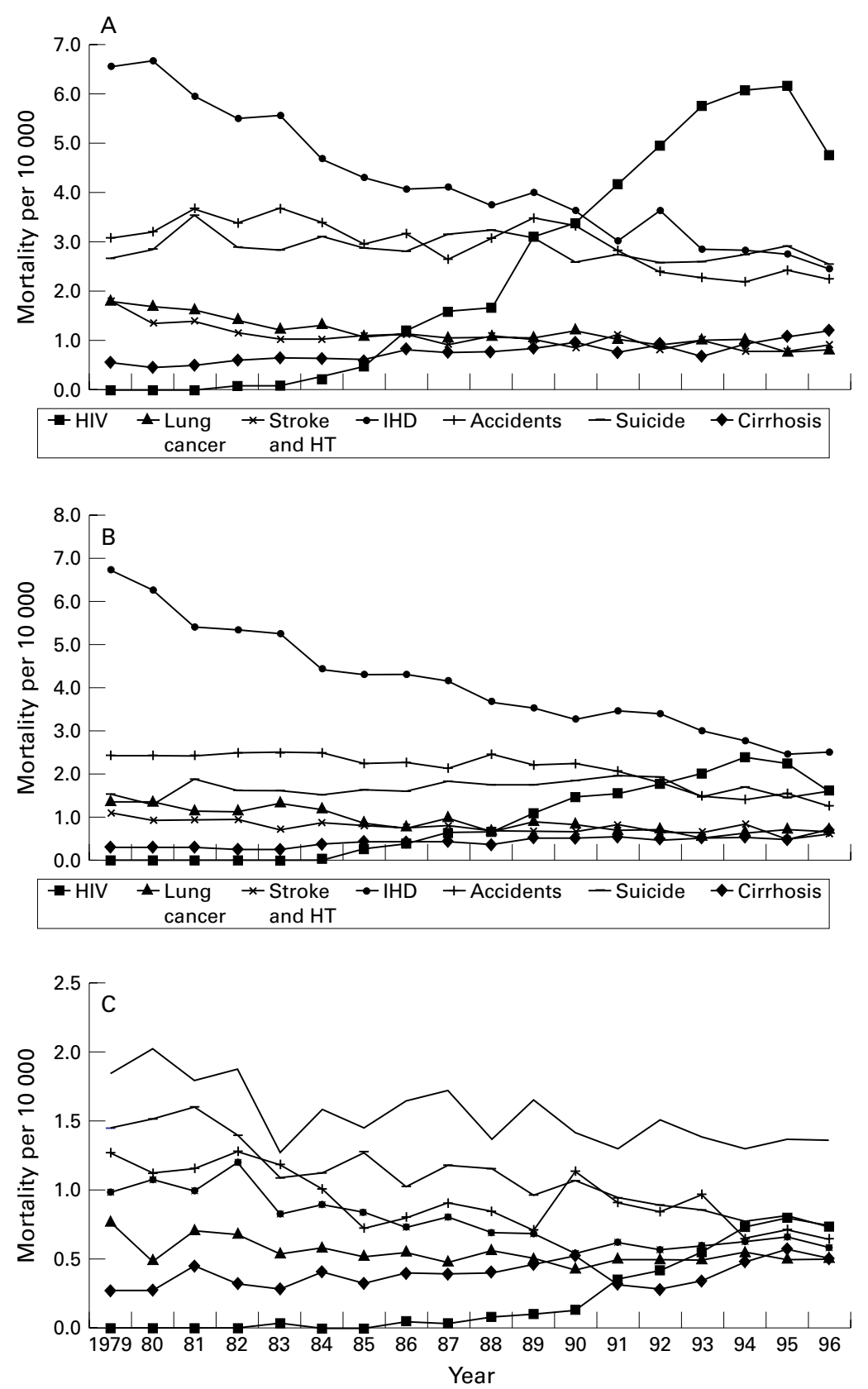

\begin{tabular}{|c|c|c|c|c|c|}
\hline$\rightarrow$ & $\begin{array}{l}\text { - Lung } \\
\text { cancer }\end{array}$ & $\rightarrow-I H D$ & $+\mathrm{Acc}$ & - Suicide & $\begin{array}{r}\text { Breast } \\
\text { cancer }\end{array}$ \\
\hline
\end{tabular}

Figure 3 Mortality by cause. (A) Inner London males aged 15-54, (B) outer London males ages 15-54, (C) inner London females aged 15-54.

of England and Wales as the standard at 100) for inner London increased significantly from 1983-5 onwards from 127.8 (95\% CI 124.4$131.1)$ to 171.3 (95\% CI $167.0-175.7)$ in 1994-6. When HIV related deaths were excluded from the observed deaths, the SMRs showed no significant increase during this time.

Figure 2B shows a similar analysis for outer London, which typically has lower mortality than the rest of England (see fig 1). The SMR increases steadily from 88.7 (95\% CI 86.7$90.9)$ in $1979-81$ up to 100.7 (95\% CI 98.2-103.3) in 1994-6. When HIV related deaths were excluded from the outer London figures, there was no overall increase and between 1994 and 1996 there had been a small decline in relative mortality compared with the rest of England and Wales (excluding HIV).
Figure 3A shows that HIV (adjusted for underreporting and change in place of residence) has been the leading cause of death in adult men aged 15-54 in inner London since 1990: above suicide, ischaemic heart disease (IHD), accidents, cirrhosis, cerebrovascular disease, and lung cancer. Adjusted HIV related mortality was highest in 1995 at 6.2 per 10000 and then dropped to 4.7 per 10000 in 1996. Despite the fall in 1996, adjusted HIV associated mortality in inner London was substantially higher than the second commonest cause of death: IHD at 2.4 per 10000 in 1996.

In males aged 15-54 in outer London (fig $3 \mathrm{~B})$, the adjusted estimates suggest that HIV was the second leading cause in adult men: above lung cancer, stroke, and cirrhosis. HIV related deaths in this age group were highest in 1994 at 2.4 per 10000 compared with 2.7 for IHD, 1.7 for suicide and undetermined, and 1.3 for accidents.

Figure 3C shows trends in the main causes of death in adult women aged 15-54 in inner London. By 1996, HIV infection was the second leading cause of death, at 0.74 per 10000 in women (15-54), behind breast cancer at 1.36 per 10 000. Even in outer London, as a cause of death HIV was still important at 0.42 per 10000 in 1996 on par with premature mortality from lung cancer and suicide.

AIDS related mortality in adult women did not fall as substantially as mortality among males. Between 1995 and 1996 (unadjusted) AIDS deaths fell by $22 \%$ (91) in males in inner London and by $27 \%$ (70) in males in outer London; in comparison, among women (unadjusted) AIDS deaths fell by $6 \%$ (3) in inner London and increased by $43 \%$ (12) in outer London.

\section{Discussion}

In inner London the downward trend in mortality among adult men has been reversed in the past decade, in contrast with the rest of England and Wales. Our data suggest that HIV infection was largely responsible for this reversal. There are many factors that will affect geographical variation in all cause mortalityfor example, aging, ethnic groups, and changes in the relative levels and distribution of deprivation that also are associated with London. However, among adult males in London these would appear to be eclipsed by the HIV epidemic. Clearly, analyses that seek to explain geographical differences in mortality without taking into account the impact of HIV in London may lead to erroneous conclusions, such as suggesting that deprivation alone is the cause of health inequalities. ${ }^{17}$ Among women, the effects of HIV on trends in all cause mortality were not as clear.

HIV infection, however, is not just a cause of an increase in "excess" mortality but is the leading cause of premature mortality among men aged 15-54 in inner London from 1990 onwards and by far the largest cause in 1996. It was also close to becoming the second commonest cause of premature mortality in women aged 15-54 in 1995 and 1996. Similar analyses have shown HIV infection to be a 
major cause of mortality among Italian women. ${ }^{18}$ The substantial fall in HIV related mortality in 1996 among males did not alter the position of HIV compared with other causes of death. The decline in HIV related mortality did not occur to the same degree among women dying from HIV causes, though the numbers were too small to speculate on why there was a difference between males and females.

The descriptive epidemiology reported here provides the first strong evidence of the impact of HIV on mortality within a significant population within England and Wales: inner London that has a population of over 1.5 million ( $5 \%$ of the total) aged $15-54$. These analyses are important and should be regularly updated. Firstly, the data are a stark reminder of the continued need for resources in HIV prevention activities. ${ }^{4}$ Secondly, they show trends in deaths before and at the beginning of the use of combination therapy and, therefore, provide a background against which its success in preventing progression to AIDS and death can be measured. ${ }^{16}$ In particular, they provide policymakers with a clear comparison of HIV related mortality against other leading causes of premature mortality which surveillance data alone sometimes fail to give. Thirdly, they confirm that HIV is a major public health problem in London and that public health priorities are clearly different in London from the rest of the country. Finally, they emphasise the value of using surveillance and mortality statistics together in order to correct otherwise misleading analyses, and to identify the true scale of the HIV epidemic. Further studies will extend the record linkage between AIDS reports and ONS death certificates to enable better interpretation of mortality data and monitoring of the impact of HIV.

We thank PHLS CDSC for providing the data, in particular Nei McDonald, Janet Mortimer, and Dr Barry Evans.
Funding: North Thames Regional Office, and Department of Health Unlinked Anonymous Screening Programme. Conflict of interest: None.

Contributors: $\mathrm{MH}$ initiated the study and wrote the first draft; $\mathrm{MB}$ and DD provided additional analyses and interpretation and HW participated in the interpretation and editing of the paper.

1 Murray CJL, Lopez AD. Mortality by cause for eight regions of the world: Global Burden of Disease Study. Lancet 1997;349:1269-76.

2 The changing global epidemiology of HIV infection and AIDS. In: The epidemiology of HIV infection and AIDS. Commun Dis Rev 1997;7:R134-6.

3 Gregson S, Garnett GP, Anderson RM. Is HIV-I likely to become a leading cause of adult mortality in sub-Saharan Africa. F Acquir Immun Defic Syndr 1994;7:839-52.

4 Coates TJ, Aggleton P, Gutzwiller F, et al. HIV prevention in developed countries. Lancet 1996;348:1143-8.

5 Selik RM, Chu SY, Beuhler JW. HIV infection as leading cause of death among young adults in US cities and states. 7AMA 1993;269:2991-9.

6 Conti S, Farachi G, Prati S. AIDS as a leading cause of death among young adults in Italy. Eur $\mathcal{F}$ Epidemiol 1994;10:669-73

7 Casabona J, Blanch C, Vall M, et al. Pramture mortality related to AIDS among men and women in Catalonia. AIDS 1993;7:1099-103.

8 Department of Health. On the state of the public health: annual report of the Chief Medical Officer of Department of Health for the year 1992. London: HMSO, 1993:36-7.

9 Aldous J, Hickman M, Ellam A, et al. Impact of HIV infection on mortality in young men in a London health authority. BMF 1992;305:219-21.

10 McCormick A. Unrecognised HIV related deaths. BMF 1991;302:1365-7.

11 King MB. AIDS on the death certificate:the final stigma BMF 1989;289:734-6.

12 Charlton J. Which areas are healthiest? Population Trends 1996;83:17-24.

13 Bardsley M, Basnett I, Congdon P, et al. Changing mortality rates in London: a review of the evidence. London: Health of Londoners Project, 1997.

14 Hogg RS, O'Shaughnessy MV, Gataric N, et al. Decline in deaths from AIDS due to new antiretrovirals. Lancet 1997; 349:1294.

15 Day NE, chairman. The incidence and prevalence of acquired immune deficiency syndrome and other severe HIV disease in England \& Wales for 1995-1999:
projections using data to end of 1994. Commun Dis Rev 1996;6:1-24

16 Hickman M, Aldous J, Gazzard B, et al. AIDS surveillance: a direct assessment of under-reporting. AIDS 1993;7: 1661-5.

17 Raleigh VS, Kiri VA. Life expectancy in England: variations and trends by gender, health authority, and level of deprivation. I Epidemiology Community Health 1997;51: deprivation.

18 Conti S, Lepri AC, Facrchi G, et al. AIDS: a major public health problem among young Italian women. AIDS 1996;10:407-11. 